\title{
¿Pertinencia? del principalismo para la ética de la investigación biomédica regional en tiempos de la pandemia Covid-19
}

DOI: https://doi.org/10.33262/ap.v3i4.1.122

\begin{abstract}
(c) (i) (5)
ar HC EM

Relevance? of principlism for the ethics of regional biomedical research in times of the Covid-19 pandemic
\end{abstract}

Ingrid González López. ${ }^{1}$

\begin{abstract}
The Covid-19 pandemic constitutes a challenge for humanity. This has become an exceptional catalyst in the debates around the ethics of biomedical research that needs to go with the scientific-investigative processes, indispensable today, in the search for solutions to the state of epidemiological catastrophe experienced by humanity. For the countries of Our America, these debates acquire the greatest relevance/specificity, since on many occasions, less global and authentic emergencies have led to the implementation of international, unilateral, and abusive biomedical research. It is in this context that it is surprising that the Pan American Health Organization has issued a regulatory instrument as Guidance for the ethical supervision of COVID-19 research in the face of the generation of new evidence (2020), which is profoundly principlist. The objective of this article is: to analyze the relevance? of principlism for the ethics of regional biomedical research in times of the current pandemic. Above the recognition of the value that for this field and context they have: the protection of social vulnerability, the opposition to a double ethical standard to evaluate the moral validity of controlled clinical trials, the moral responsibility of the sponsors towards the research subjects a once the study is concluded, and the centrality of solidarity for research with foreign "cooperation"; four precepts, not in vain, already identified in CIOMS-WHO Guidelines (2002).
\end{abstract}

\footnotetext{
${ }^{1}$ Universidad de La Habana, Facultad de Filosofía, Historia y Sociología. Ciudad de La Habana, Cuba. ingred@ffh.uh.cu
} 
Keywords: Principlism, ethics of biomedical research, social vulnerability, double ethical standard, responsibility, solidarity

\section{Resumen}

La pandemia Covid-19 constituye un desafío para el género humano. Esta se ha convertido en un catalizador excepcional de los debates en torno a la ética de la investigación biomédica que precisa acompañar a los procesos científico-investigativos, indispensables hoy, en la búsqueda de soluciones al estado de catástrofe epidemiológica vivenciado por la humanidad. Para los países de Nuestra América, estos debates adquieren la mayor relevancia/especificidad, pues en no pocas ocasiones, urgencias menos globales y auténticas han dado lugar a la implementación de investigaciones biomédicas internacionales, unilaterales y abusivas. Es en este contexto donde sorprende que la Organización Panamericana de la Salud haya emitido un instrumento regulatorio como Orientación para la supervisión ética de las investigaciones sobre COVID-19 ante la generación de nueva evidencia (2020), profundamente principalista. El presente artículo se propone como objetivo: analizar la ¿pertinencia? del principalismo para la ética de la investigación biomédica regional en tiempos de la actual pandemia. Arriba al reconocimiento del valor que para este campo y contexto tienen: la protección de la vulnerabilidad social, la oposición a un doble estándar ético para evaluar la validez moral de ensayos clínicos controlados, la responsabilidad moral de los patrocinadores para con los sujetos de pesquisa una vez concluido el estudio, y la centralidad de la solidaridad para la investigación con "cooperación" extranjera; cuatro preceptos, no en balde, ya identificados en Pautas CIOMS-OMS (2002).

Palabras claves: Principalísimo, ética de la investigación biomédica, vulnerabilidad social, doble estándar ético, responsabilidad, solidaridad.

\section{Introducción}

Dentro de la línea de la bioética entendida como ética aplicada -inaugurada por Andrés Hellegers, en 1971, como director fundador de The Joseph and Rose Kennedy Institute for the Study of the Human Reproduction and Bioethics de la Universidad de Georgetownel principalismo constituye una propuesta teórico-metodológica, ciertamente influyente. Esta afirmación, puede ser constatada con relativa facilidad, a través de la expresión de "Mantra de Georgetown", ampliamente empleada por bioeticistas contemporáneos para referirse al libro que da origen al principalismo. Aunque la expresión no está exenta de legítima ironía, no por ello consigue dejar de insinuar el rol -cuasi absoluto- que desempeñó este modelo de análisis y solución a los problemas y conflictos de valores en el ámbito de la práctica clínica y la investigación biomédica, durante las primeras décadas de vida de la línea, e incluso, del saber en cuestión.

Sin pretender anatemizar al principalismo, sorprende que hoy, la Organización Panamericana de la Salud (OPS) haya emitido un documento como Orientación para la 
supervisión ética de las investigaciones sobre COVID-19 ante la generación de nueva evidencia (2020), de letra y fundamentación, profundamente principalista. Ante este hecho y sus posibles consecuencias, el presente artículo se propone como objetivo: analizar la ¿pertinencia? del principalismo para la ética de la investigación biomédica regional en tiempos de la actual pandemia. Para ello, tiene a bien, concebir su desarrollo compuesto por tres partes integrantes: 1) El qué del principalismo; 2) Ventajas y limitaciones generales de la metodología principalista; y 3) Sobre el principalismo y los riesgos de una historia desestimada en la ética de la investigación biomédica regional en tiempos de la pandemia Covid-19. La última de ellas, encuentra como principal referente de comparación/contrastación a las Pautas éticas internacionales para la investigación biomédica en seres humanos (2002). En este caso, el criterio de selección empleado no responde a una vigencia normativa -en efecto, hoy suplantada con la emisión de las Pautas éticas internacionales para la investigación relacionada con la salud con seres humanos (2017)- sino al reconocimiento de su valor testimonial y el sentido de memoria histórica que la misma representa.

Metodología: Se realiza un estudio cualitativo que se apoya de manera fundamental en la revisión bibliográfica, a partir de la consulta de las fuentes primarias y secundarias de información. En las primeras figuran un libro y dos documentos normativos insustituibles: Principles of Biomedical Ethics (2001), Pautas éticas internacionales para la investigación biomédica en seres humanos (2002) y Orientación para la supervisión ética de las investigaciones sobre COVID-19 ante la generación de nueva evidencia (2020). En las segundas, se encuentra el resto de los textos que se citan a lo largo del cuerpo del presente artículo y declaran en el apartado final de referencias bibliográficas; procurando privilegiar el estudio de autores, obras, artículos y revistas de relevancia para el tema, y en la medida de lo posible, que fueran de la región. En el orden teórico, se emplearon los métodos de análisis y síntesis de la información recopilada, y de forma especial, la técnica comparativa entre la metodología principalista (objeto de crítica, no en abstracto sino en correspondencia al campo/contexto potencial de aplicación, definidos en el objetivo del artículo) y el contenido de las Pautas éticas internacionales para la investigación biomédica en seres humanos (2002).

\section{Resultados}

\section{El qué del principalismo}

El principalismo tiene un antecedente histórico inmediato, que es preciso reconocer. Este es el Belmont Report; cuyo proceso de génesis se remonta al año 1974, con la aprobación presidencial de la National Research Act y la creación, por parte del Congreso estadounidense, de la National Commission for the Protection of Human Subjects of Biomedical and Behavioral Research. Ambos acontecimientos -marcados por el conocimiento público del estudio Tuskegee (Pence, 1995, p. 225) y, en consecuencia, el evidente estado de conmoción general vivenciado por la sociedad norteña - respondían a la necesidad inminente de establecer nuevas directrices, de alcance federal, en materia de regulación de la investigación científica doméstica. En particular, a la comisión le fue 
encargada la tarea de identificar aquellos principios éticos básicos indispensables a tener en cuenta en las investigaciones que incluyeran seres humanos, para evitar casos de abuso flagrante como el del recién cerrado estudio. El informe, publicado en 1978, constituyó el resultado de los 4 años de trabajo de dicha comisión.

En 1979, Tom L. Beauchamp, filósofo utilitarista e investigador en el Kennedy Institute of Ethics de la Universidad de Georgetown -quien había formado parte de la Comisión Nacional y participado en la elaboración del Belmont Report- y James F. Childress, deontólogo cristiano y profesor en el Departamento de Estudios Religiosos en la Universidad de Virginia, publican el libro que patenta el nacimiento del principalismo: Principles of Biomedical Ethics. En esta obra -llamada a convertirse en un clásico de la literatura bioética, con seis ediciones hasta el momento (1979, 1983, 1989, 1994, 2001 y 2009)- Beauchamp y Childress lanzan su propuesta teórico-metodológica, que a diferencia del Belmont Report: amplía el campo de aplicación del sistema de principios a todo el ámbito biomédico (práctica clínica e investigación); reformula el principio de respeto a las personas como el de respeto a la autonomía, propiamente; distingue la beneficencia de la no maleficencia; introduce una regla general (los cuatro principios son prima facie); y establece la especificación y la ponderación de los principios como procesos que garantizan el razonamiento moral en la toma de decisiones.

El primero de los principios que aparece en Principles es el de respeto a la autonomía. En su denominación, es el único que presenta una estructura gramática, preciso atender. Para los autores no se trata de la autonomía a secas, sino del respeto a la autonomía. Esto justifica dos distinciones esenciales, realizadas por Beauchamp y Childress, para definir el entorno conceptual del principio en cuestión. La primera es la distinción entre personas y acciones autónomas; que en consecuencia, les permite centrar la atención en la caracterización de esta última. De ahí su conocido planteamiento de las tres condiciones para evaluar la autonomía de las acciones humanas: intencionalidad, comprensión y libertad de controles externos; el reconocimiento de que la autonomía de las acciones no es completa, si no que admite grados; y en consecuencia, la propuesta de un criterio de substancialidad -en específico, con respecto a la comprensión y a la libertad de controles externos- como condición suficiente para afirmar que el agente ha actuado autónomamente (Ferrer, 1998, p. 42).

La segunda distinción esencial, otorga pleno sentido a la estructura gramatical que da título a este principio. Con ella, los autores rebasan ya el marco del análisis conceptual, indispensable, pero fragmentado por específico y se adentran como tal, en el terreno del contenido normativo del propio principio. Es la distinción que Beauchamp y Childress plantean entre las acciones y las actitudes respetuosas, no ya del agente moral, cual Robinson Crusoe solo en una isla, si no las de sus interlocutores, pues es en el plano de las relaciones interpersonales donde las acciones autónomas se ponen a prueba. En correspondencia a la formulación del principio, es evidente que para los autores, el respeto por la autonomía ajena no se reduce a una cuestión de meras actitudes respetuosas. En palabras de Beauchamp y Childress: 
Respetar un agente autónomo es, como mínimo, reconocer el derecho de la persona a sostener puntos de vista, a realizar elecciones, y emprender acciones basadas en los valores y las creencias personales, Dicho respeto incluye la acción respetuosa, y no meramente la actitud respetuosa. También requiere algo más que la no interferencia en los asuntos personales de los demás. Incluye, al menos en algunos contextos, la obligación de fortalecer y sostener la capacidad de otros para una elección autónoma, mientras se ayuda a disipar temores y otras condiciones que destruyen o perturban sus acciones autónomas. El respeto, en estos casos, involucra el reconocimiento de los derechos a tomar decisiones, y posibilitar la actuación autónoma, mientras que el irrespeto a la autonomía involucra actitudes y acciones que ignoran, insultan o degradan los derechos de otros a la autonomía. (Beauchamp \& Childress, 2001, p. 63)

El segundo de los principios que aparece en la obra es el de la no maleficencia (no hacer daño a los demás intencionadamente). Este, data de una larga tradición en el campo de la ética médica. Así, junto a la beneficencia (obligación moral de actuar en beneficio de los otros) constituye uno de los dos principios rectores del Juramento Hipocrático y en el Código de Percival -de referencia mundial en la segunda mitad del siglo XIX-se convierte en la máxima principal de la conducta del médico hacia el paciente: primero no hacer daño (primum non nocere). En Principles, si bien Beauchamp y Childress reconocen que no en todos los casos el ejercicio de delimitar los entornos de la no maleficencia y de la beneficencia está exento de dificultad, advierten la necesidad de plantearlos como principios distintos. Al respecto, señalan tres características generales de las reglas morales basadas en este principio: "1) imponen prohibiciones negativas, 2) se deben obedecer imparcialmente, y 3) pueden dar pie para establecer prohibiciones sancionadas por la ley" (Ferrer, 1998, p. 47). Su mayor especificidad, en el sistema propuesto por Beauchamp y Childress, se concreta con la formulación del principio como prima facie.

La beneficencia es el tercer principio abordado en Principles. A diferencia de la ética médica tradicional, el principalismo exige que no sea ejercida a ultranza por el especialista (médico o investigador), si no teniendo en cuenta los deseos y voluntad del usuario de estos servicios (paciente o sujeto de pesquisa), de conformidad con su propio sistema de valores. Para su análisis conceptual, Beauchamp y Childress, plantean dos distinciones importantes. La primera es la establecida entre la beneficencia general (que obliga a actuar beneficentemente con los demás) y la beneficencia específica (que obliga a actuar beneficentemente con aquellos que se tienen relaciones especiales). Afirman que, en el plano de las relaciones especiales, los actos de beneficencia tienen carácter obligatorio y no son meros ideales morales. La segunda distinción versa sobre dos dimensiones inherentes a la beneficencia, así entendida: la formulada entre la beneficencia positiva (que apela a su definición básica, es decir, la obligación moral de realizar acciones positivas para beneficiar a los otros) y la utilidad (que obliga a ponderar los riesgos y los beneficios). Los autores advierten que ambas dimensiones de la beneficencia se complementan entre sí; y en particular, son de gran interés en el terreno 
de la biomedicina, donde las intervenciones biomédicas están sujetas a riesgos, efectos adversos y tienen contraindicaciones.

La justicia constituye el último de los principios -de acuerdo con el orden en que son presentados en la obra. Al igual que el primero de ellos, carece de una historia previa, en los marcos de la ética médica tradicional. Más es un concepto central en numerosas teorías políticas, cuyo tratamiento los autores no desprecian; en particular, los casos de las teorías: igualitarias, comunitarias, libertarias y utilitarias. En Principles, la justicia distributiva (la búsqueda de solución al agudo problema de la distribución equitativa de las cargas y los beneficios en la sociedad) constituye el centro de atención de Beauchamp y Childress. En este sentido, no adoptan la posición de conceder más valor, a priori, a una teoría específica en detrimento del resto. Los autores reconocen, que es a la sociedad concreta, a la que le corresponde efectuar semejante elección. El principio de justicia, entendido desde la perspectiva de la justicia distributiva, lo que advierte es la necesidad de pensar en los derechos de las terceras partes involucradas, el bien común. Y llega a proponer la defensa de un "mínimo decente", frente a la lógica ampliamente extendida de resolución de los problemas de equidad a través del análisis de la relación costo-beneficio, interpretado en clave economicista.

En términos de metodología propiamente, el principalismo se destaca por proponer una regla general que condiciona una singularidad per se a cada principio, y a la vez, dota al sistema de la cohesión flexible que lo caracteriza. Esta regla general consiste en la definición de sus principios en tanto prima facie. Con dicha definición los autores plantean que los cuatro principios (respeto a la autonomía, no maleficencia, beneficencia y justicia) a priori tienen igual jerarquía, obligan siempre más no para siempre, tienen carácter vinculante pero este está abierto a la excepción. Como tal, la concepción de esta regla tiene por fuente de inspiración, la distinción entre deberes prima facie (prima facie duties) y deberes de hecho (actual duties) formulada por W. D. Ross, en el libro The Right and the Good. Esta distinción, replanteada por los autores de Principles en términos de obligaciones prima facie y de hecho, constituye el soporte teórico de su regla general. $\mathrm{Al}$ respecto, Beauchamp y Childress plantean:

Una obligación prima facie debe cumplirse, excepto si, en una situación particular, entra en conflicto con una obligación de igual o de mayor valor. Este tipo de obligación siempre compromete, a no ser que una obligación moral concurrente sea más importante y esté por encima de la primera en una situación concreta. (Beauchamp \& Childress, 2001, p. 14)

Así, en el principalismo, solo es obligación de hecho, aquella obligación moral que demuestre tener "mayor valor" o "importancia" en un caso concreto.

Otros dos elementos metodológicos esenciales de esta propuesta lo constituyen la especificación y la ponderación. Ambos elementos aseguran el razonamiento moral en los procesos de toma de decisiones en el ámbito biomédico. El primero de ellos, la especificación, como su nombre mismo lo indica advierte sobre la necesidad de concretar o "especificar" -dotar de contenido específico- a cada principio, planteado de forma aún 
demasiado general en la propuesta en cuestión. Beauchamp y Childress plantean que la especificación de los principios generales a normas menos indeterminadas, de conformidad con las exigencias planteadas por el caso objeto de análisis, constituye la piedra angular del propio proceso deliberativo.

Sin embargo, la especificación no siempre resulta suficiente. En no pocas ocasiones el proceso deliberativo se tiene que enfrentar a una situación de conflicto entre los principios o las normas particulares concretas, que exceden la competencia de la especificación. Por ello, los autores también recomiendan la ponderación. Ponderar significa sopesar o poner en la balanza los principios prima facie para determinar cuál de ellos es el principio de hecho, es decir, cuál de los principios o normas que entran en conflicto tiene mayor importancia o peso relativo y, por tanto, acusa precedencia en el caso concreto. Es aquí, donde el sistema de Beauchamp y Childress devela su esencia genuinamente utilitarista. La ponderación exige la formulación de un criterio para poder decidir con calidad moral; los autores de Principles adoptan un criterio de carácter teleológico o consecuencialista: el principio o norma preponderante será aquel que produzca las mejores consecuencias posibles para los sujetos involucrados.

\section{Ventajas y limitaciones generales de la metodología principalista}

Si bien el principalismo ha sido la tendencia más influyente del grupo de Georgetown, no menos verídico es el hecho, que en el devenir de su trayectoria -por contextos culturales y socio-económicos diversos- este modelo teórico-metodológico también ha recibido varias críticas; las que a la larga, han terminado por contribuir al proceso de enriquecimiento y maduración de la bioética entendida como ética aplicada. En este sentido, sobresalen dos cuestionamientos, relacionados entre sí. No pocos autores coinciden en reconocer como una debilidad de esta propuesta, la ausencia de un orden jerárquico entre los principios, y a la vez, cierta tendencia hacia la exaltación del individualismo.

La primera de estas críticas, tiene por fundamento último, la tesis de que los cuatro principios -ciertamente- representan niveles distintos de la moralidad. Así, el respeto por la autonomía y la beneficencia acusan el nivel individual o privado, y la no maleficencia y la justicia advierten el nivel público o social. Por tanto, los autores plantean que en situaciones de conflicto entre principios de niveles de moralidad distintos (privado y público o individual y social) no basta con la regla general y el criterio de la ponderación, ofrecidos por Beauchamp y Childress. Concurren en señalar, que este tipo de conflicto hallaría solución satisfactoria con el establecimiento de un ordenamiento jerárquico de los principios, donde los principios de no maleficencia y justicia tengan mayor fuerza vinculante que los de respeto a la autonomía y beneficencia. Esta crítica y su propuesta de solución para el manejo de conflicto entre principios de niveles de moralidad distintos, no ha sido aceptada por los autores de Principles. Es válido destacar que su reconocimiento podría redundar en una pérdida de la ductilidad que ha sido tenida como un mérito indiscutible de esta propuesta. 
En cuanto a la segunda crítica -su tendencia hacia la exacerbación del individualismohalla fundamento, en los resultados de la aplicación de esta metodología, en el ámbito biomédico norteamericano, propiamente. Es cierto que, en los Estados Unidos, la aplicación del principalismo a situaciones de problemas y conflicto de valores morales en la práctica clínica y la investigación acusa una marcada preferencia por el respeto a la autonomía y las cuestiones concernientes al procedimiento para hacerlo efectivo, el consentimiento informado. En la quinta edición de Principles -la del 2001- los autores han negado que este cuestionamiento tenga validez. Bien vistas las cosas, la preferencia evidenciada en la sociedad norteña por principios del nivel privado en lugar de principios del nivel público de la moralidad acusa una tendencia social y no una debilidad per se del modelo. Por el contrario, la apertura hacia el contexto en materia de deliberación moral que en el sistema de Beauchamp y Childress es una consecuencia de la regla general de definir los principios en tanto prima facie-constituye otra de las fortalezas que distinguen al principalismo de la ética médica tradicional.

En síntesis, el principalismo constituye uno de los modelos teórico-metodológicos principales de la bioética entendida como ética aplicada. Su influencia en esta línea del saber en cuestión, más allá de los mimetismos iniciales, ha sido relevante. Entre sus aciertos principales destacan: la gran ductilidad que ha demostrado tener este sistema para la deliberación moral en el campo biomédico; la exigencia de transitar hacia modelos de relación especialistas-usuarios de estos servicios (médico-paciente e investigador-sujeto de pesquisa) más horizontales, simétricos; y su probada efectividad para tratar dilemas y conflictos morales en situaciones límites diversas de la práctica clínica, y en materia de ética de la investigación. Más, en estos propios predios, la propuesta de Beauchamp y Childress también presenta limitaciones. A modo de ejemplo se puede mencionar su inefectividad emblemática para abordar problemas éticos característicos de la atención primaria de salud, con su consecuente indiferencia hacia las acciones de prevención, promoción y educación en salud. Además, cuando los dilemas y conflictos biomédicos rebasan el marco de las relaciones interpersonales e involucran a la sociedad como un todo, este modelo tiende a fallar.

Sobre el principalismo y los riesgos de una historia desestimada en la ética de la investigación biomédica regional en tiempos de la pandemia Covid-19

El advenimiento de la actual pandemia -causada por el SARS-CoV-2, un virus desconocido en la historia de la epidemiología humana, con un alto poder infectocontagioso y elevada morbi-mortalidad selectiva: personas de la tercera edad, enfermos crónicos y pobres- ha intensificado los debates en torno a la ética de la investigación biomédica (Acosta, 2020; Helge et al., 2020). Teniendo en cuenta las bondades, y sobre todo, las limitaciones generales del principalismo -antes analizadas- sorprende que la OPS haya emitido un documento como Orientación para la supervisión ética de las investigaciones sobre COVID-19 ante la generación de nueva evidencia (2020), de letra y fundamentación, profundamente principalista. 
No es necesario salir de la propia concepción de la bioética entendida como ética aplicada, y dentro de ella, de la rama dedicada al estudio y desarrollo de la ética de la investigación que involucra a seres humanos, para encontrar soporte y directrices normativas de mayores niveles de pertinencia hoy, en el contexto global-regional de la pandemia COVID-19. Este campo cuenta con una historia ganada, que nos es posible desconocer, máximo por una entidad como la OPS, llamada a velar por la satisfacción de las complejas necesidades en materia de salud de la región. Dicha historia demuestra con suficiente elocuencia, que en Nuestra América, el principalismo se revela como insuficiente porque queda desbordado por la presencia de un conflicto de fondo: el producido entre problemas emergentes y problemas persistentes.

La COVID-19 representa un desafío para el género humano. Es innegable que a este desafío la humanidad tiene que buscar salida y hacerlo de forma mancomunada. Sin embargo, no es posible encontrar respuestas al margen del reconocimiento de las condiciones de profunda desigualdad imperantes en el mundo, que lejos de ser atenuadas en el contexto de la actual pandemia estas amenazan con robustecerse hasta límites dantescos. El campo de la ética de la investigación no es ajeno a esta alarmante realidad. De ahí, la extrema importancia de pensar en el ethos ético que ha de guiar las pesquisas biomédicas que se desarrollen en nuestra región. Respeto a la autonomía, no maleficencia, beneficencia y justicia no son los únicos principios que se precisan defender. Sin negar su valor, la protección de la vulnerabilidad social, la denuncia y oposición a un doble estándar ético para evaluar la validez moral de los ensayos clínicos controlados, la responsabilidad moral de los patrocinadores externos para con los sujetos de pesquisa (individuos y comunidades) una vez concluido el estudio, y la centralidad del principio de solidaridad para la investigación con "cooperación" extranjera - no en balde, ya identificados en un documento normativo internacional como Pautas éticas internacionales para la investigación biomédica en seres humanos (2002)- denotan mayor relevancia en la región.

Este último documento mencionado, también es conocido como Pautas CIOMS-OMS teniendo en cuenta a las dos entidades rectoras del proyecto en cuestión (el Consejo de Organizaciones Internacionales de las Ciencias Médicas y la Organización Mundial de la Salud. Hasta la fecha, cuenta con cuatro versiones (1982, 1993, 2002 y 2017). A favor de su pertinencia en el ámbito de la ética de la investigación a nivel local, baste recordar que desde el inicio -a fines de la década del 70- el proyecto se planteó como objetivo explícito:

Preparar pautas "para indicar el modo en que los principios éticos que debieran guiar la conducta de la investigación biomédica en seres humanos, establecidos por la Declaración de Helsinki, podían ser aplicados en forma efectiva, especialmente en los países en desarrollo, considerando sus circunstancias socioeconómicas, sus leyes y regulaciones, así como sus disposiciones ejecutivas y administrativas". (OPS \& CIOMS, 2002, p. 11)

Las versiones de 1993, 2002 y 2017 fieles a este propósito originario, han logrado ofrecer pautas enriquecidas, no solo como resultado de un trabajo "de mesa" o puramente formal, 
sino de cara al diálogo obligado con los desafiantes nuevos derroteros marcados por la propia investigación biomédica in situ. En este sentido, las Pautas CIOMS-OMS (2002) no constituyen una excepción. Ante dos controversiales ensayos clínicos sobre el VIH/SIDA, promovidos por el Instituto Nacional de Salud de los Estados Unidos en países subdesarrollados -a finales de la década del 90- tienen el mérito histórico de haber introducido una serie de disposiciones específicas en torno a preceptos normativos de máxima prioridad y vigencia para la investigación biomédica internacional que se desarrolle en la región.

La protección de la vulnerabilidad social tiene una cita especial en Pautas CIOMS-OMS (2002). Como tal, es un concepto de corta data, inexistente en el modelo teóricometodológico principalista. Beauchamp y Childress cuanto más llegan a hablar de vulnerabilidad biológica (niños, ancianos, enfermos mentales y pacientes en coma) asociada al análisis del principio de respeto a la autonomía (autonomía comprometida) y del procedimiento para hacerlo efectivo, el consentimiento informado (a manos de representantes válidos o legales). Más, no de vulnerabilidad social.

Esta forma de vulnerabilidad, que proviene de reflexiones propias de las Ciencias Sociales es asumida en el campo de la ética de la investigación como "el límite de la autodeterminación y el aumento significativo de la exposición a riesgos, provocada por una situación de exclusión social" (Garrafa, 2015, p. 178). Las Pautas CIOMS-OMS (2002) reconoce tanto el concepto como la necesidad de protección de este tipo de sujetos, participantes potenciales de pesquisas biomédicas en la región. A modo de argumentación, valga apuntar que su tratamiento está incluido en 5 de sus 21 pautas ( 3 , 10, 12, 20 y 21); y de forma central en la 10, titulada Investigación en poblaciones y comunidades con recursos limitados. En su conjunto, contribuyen a la implementación de procedimientos para disminuir los riesgos asociados a la inclusión de estas poblaciones en grupos muestrales, y a su vez, a garantizar una distribución más justa de los beneficios resultantes de cualquier intervención realizada sobre las mismas.

La posibilidad de un doble estándar ético para evaluar la validez moral de los ensayos clínicos controlados tiene por detonante principal pocos, pero significativos cambios operados en la Declaración de Helsinki en el siglo XXI (tres "revisiones": Edimburgo 2000, Seúl 2008, Fortaleza 2013; y dos "alteraciones": Washington 2002 y Tokio 2004) (Garrafa, 2015, p. 175). De modo neurálgico, la cuestión de semejante nivel de relajamiento "ético" en el instrumento normativo más socorrido en este campo a nivel mundial se concentra en el empleo del adjetivo current, introducido en la Declaración de Helsinki (2000) para calificar al tipo de tratamiento permitido del grupo control. Nótese que el adjetivo que utiliza la traducción al idioma español de dicha versión es el de “disponible". En efecto, este cambio, en apariencia "mínimo" tiene implicaciones éticas relevantes para las investigaciones biomédicas internacionales que se realicen entre países ricos y en vías de desarrollo, pues resulta evidente que "el mejor tratamiento disponible" en los países anfitriones puede llegar a ser mucho menos eficiente -e incluso inexistenteen comparación con "el mejor tratamiento disponible" en los países patrocinadores de 
estos ensayos clínicos controlados (Penchaszadeh, 2002; Fernández, 2007; Acosta, 2009; Garrafa, 2015).

Ciertamente, las Pautas CIOMS-OMS (2002) se pronuncian a favor de combatir la existencia de un doble estándar para la consideración del bienestar de los sujetos de un grupo control. A este fin, dedica su pauta más amplia, la 11 Elección del control en ensayos clínicos. En este sentido, la pauta se destaca por la defensa de dos elementos: 1) su distancia con respecto a la terminología empleada en la Declaración de Helsinki (2000) de "intervención de efectividad comprobada" en lugar de "intervención de efectividad existente"; y 2) la exposición de los tres casos en que puede ser aceptado el uso de placebo (cuando no existe una intervención de efectividad comprobada; cuando su omisión expone a los sujetos, a lo sumo, a una molestia temporal o a un retraso en el alivio de los síntomas; y cuando su uso como control no produce resultados científicamente confiables y el uso de placebo no añade ningún riesgo de daño serio o irreversible para los sujetos) (OPS \& CIOMS, 2002, p. 59). Con respecto al empleo de la expresión "el mejor tratamiento disponible" de la Declaración de Helsinki (2000), la Pauta 8 Beneficios y riesgos de participar en un estudio plantea que la disponibilidad debe ser medida a nivel global, no local (OPS \& CIOMS, 2002, p. 53).

La exigencia de responsabilidad moral de los patrocinadores externos para con los sujetos de pesquisa (individuos y comunidades) una vez concluido el estudio constituye otro precepto esencial en la ética de la investigación biomédica regional. Encuentra su correlato mayor en los derechos inalienables que asisten a estos sujetos. Más hoy, es posible advertir la implantación de cierta tendencia en las nuevas versiones de instrumentos normativos internacionales, que consiste en limitar el alcance de estos derechos en dos sentidos: 1) la temporalidad (durante la investigación) y 2) la individualidad (de los sujetos de pesquisa y no de las comunidades). Ante este hecho, las Pautas CIOMS-OMS (2002) responden con la redacción de la pauta 21 Obligación ética de los patrocinadores externos de proporcionar servicios para la atención de salud. Sin desatender los derechos en materia de atención a la salud individual de los sujetos durante la investigación, plantea: "Los patrocinadores externos tienen la obligación de poner razonablemente a disposición de la población o comunidad participante del proyecto el producto beneficioso desarrollado como resultado de la investigación" (OPS \& CIOMS, 20002, p. 86).

Por último, la centralidad del principio de solidaridad en la investigación biomédica con "cooperación" extranjera deviene una máxima para evitar las más diversas formas de explotación en los países en vías de desarrollo. A pesar de su grado de generalidad es posible identificar expresiones concretas de la misma, cuando el patrocinador de los ensayos clínicos se preocupa por: beneficiar al país anfitrión (transferencia de materiales y equipamientos modernos, tecnologías y formación de recursos humanos); tiene en cuenta las complejas necesidades en materia de salud locales y en consecuencia, el estudio se plantea contribuir a su resolución, así sea de forma parcial; y no subordina los preceptos éticos fundamentales de toda investigación biomédica a intereses científicos y/o comerciales, que solo pueden beneficiar al régimen de desigualdad imperante a nivel 
mundial. Lamentablemente, esta parece ser más la regla que la excepción. En la literatura, su expresión más extrema es conocida como investigaciones "safari" (toda la participación local se reduce a aportar información y muestras de sangre).

\section{Conclusiones}

- La protección de la vulnerabilidad social, la denuncia y oposición a un doble estándar ético para evaluar la validez moral de los ensayos clínicos controlados, la responsabilidad moral de los patrocinadores externos para con los sujetos de pesquisa (individuos y comunidades) una vez concluido el estudio, y la centralidad del principio de solidaridad para que una investigación sea con verdadera cooperación extranjera y no un asunto de mera nomenclatura constituyen cuatro preceptos normativos -sinérgicos entre sí- de elevada pertinencia para la ética de la investigación biomédica que se desarrolle en la región.

- Dichos preceptos, extraños al modelo teórico-metodológico principalista encuentran una acogida consecuente en las Pautas CIOMS-OMS (2002). Si bien es cierto que, en la tercera edición de este instrumento regulatorio internacional, sus tratamientos específicos aún presentan limitaciones; resulta indudable, que el documento en cuestión, tiene el mérito histórico de haber ofrecido un punto de partida concreto para sus desarrollos futuros.

- Estos esfuerzos deberían ser considerados por una entidad con autoridad regulatoria regional como la OPS en el contexto de la pandemia COVID-19. La urgencia de encontrar soluciones (diagnósticas, de tratamiento y prevención) al actual estado de catástrofe epidemiológica constituye un factor de riesgo excepcional para la reedición de investigaciones biomédicas de tipo abusivas en Nuestra América, que precisa ser evitado.

\section{Referencias Bibliográficas}

Acosta, J. R. (2009). Los árboles y el bosque. Texto y contexto bioético cubano. La Habana, Cuba. Publicaciones Acuario.

Acosta, J. R. (2020). Los desafíos bioéticos y biopolíticos develados por la pandemia COVID-19. Revista Cubana de Salud Pública, 46 (Supl. especial), e2629. http://www.revsaludpublica.sld.cu//index.php/spu/article/download/2629/1563

Beauchamp, T. L. \& Childress, J. F. (2001). Principles of Biomedical Ethics. New York, United States of America. Oxford University Press.

Fernández, R. (2007). La versión de la Declaración de Helsinki de Edimburgo 2000. Análisis y controversias. En Acosta, J. R. (ed.). Bioética desde una perspectiva cubana. (pp. 211-216). La Habana, Cuba. Publicaciones Acuario.

Ferrer, J. (1998). Los principios de la bioética. Cuadernos del Programa Regional de Bioética, no. 7, 37-62. 
Garrafa, V. (2015). Ética de la investigación biomédica. En Vidal, S. (Coordinadora). Manual de bioética para periodistas. (pp. 169-191). Montevideo, Uruguay. UNESCO

Helge, J., Beate, H., Holm, S. Tolo, A. K., Bjørn Hofmann B., Robertsen A., Hambro A., Shereen A., Pedersen R. \& Bernabe R. (2020). ¿Volver a qué? El papel de la ética de la investigación en época de pandemia. Revista Redbioética/UNESCO, no. $22,102-128$.

Organización Panamericana de la Salud. (2020). Orientación para la supervisión ética de las investigaciones sobre COVID-19 ante la generación de nueva evidencia. http://www.paho.org/coronavirus

Organización Panamericana de la Salud, Consejo de las Organizaciones Internacionales de las Ciencias Médicas. (2002). Pautas éticas internacionales para la investigación biomédica en seres humanos. https://www.bioética.ops-oms.org

Organización Panamericana de la Salud, Consejo de las Organizaciones Internacionales de las Ciencias Médicas (2017). Pautas éticas internacionales para la investigación relacionada con la salud con seres humanos. https://iris.paho.org/handle/10665.2/34457

Pence, G. E. (1995). Classic cases in medical ethics. New York, United States of America. McGraw-Hill.

Penchaszadeh, V. (2002). Ética de las investigaciones biomédicas en un mundo globalizado. En Acosta, J. R. (ed.). Bioética para la sustentabilidad. (pp. 439453). La Habana, Cuba. Publicaciones Acuario.

\section{LC Ciencia}




\section{PARA CITAR EL ARTÍCULO INDEXADO.}

González López, I. . (2021). ¿Pertinencia? del principalismo para la ética de la investigación biomédica regional en tiempos de la pandemia Covid-19. AlfaPublicaciones, 3(4.1), 26-39. https://doi.org/10.33262/ap.v3i4.1.122

\section{Liencia}

El artículo que se publica es de exclusiva responsabilidad de los autores y no necesariamente reflejan el pensamiento de la Revista Alfa Publicaciones.

El artículo queda en propiedad de la revista y, por tanto, su publicación parcial y/o total en otro medio tiene que ser autorizado por el director de la Revista Alfa Publicaciones.
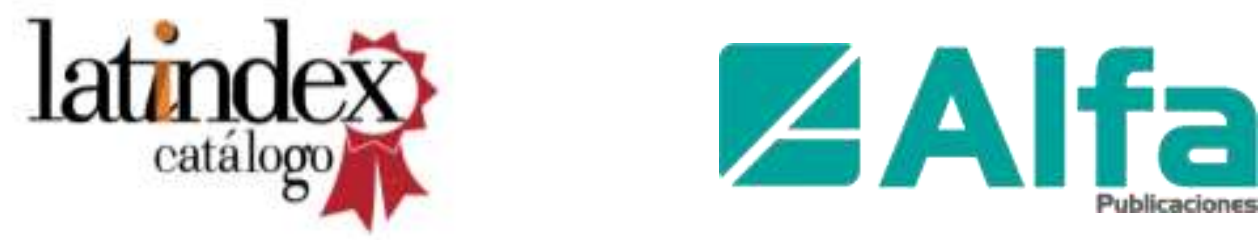